\title{
Formative Evaluation to Determine Facilitators and Barriers to Nurse-driven Implementation: Designing an Inpatient mHealth Intervention to Support Smoking Cessation
}

\author{
Amanda C. Blok, PhD MSN \\ Quantitative Health Sciences, \\ University of Massachusetts \\ Medical School \\ Amanda.Green@umassmed.edu
}

\author{
Rajani S. Sadasivam, PhD \\ Quantitative Health Sciences, \\ University of Massachusetts \\ Medical School \\ Rajani.Sadasivam@umassmed.edu
}

\author{
Thomas K. Houston, MD MPH \\ Center for Healthcare \\ Organization and Implementation \\ Research (CHOIR), \\ Bedford and Boston Veterans \\ Affairs Hospitals \\ Thomas.Houston2@va.gov
}

\begin{abstract}
The inpatient setting is often a missed opportunity for the introduction of technology to promote health using behavioral techniques. Nurses are stakeholders in the implementation of technology for patients in the inpatient setting and are essential for the determination of feasibility and relevance. The objective of this study was to identify facilitators and barriers for introduction of health-related patient technology, and specifically the appropriateness of mobile health (mHealth) technology in the hospital setting as identified by nurse leaders and staff. Methods of formative evaluation included nurse leader and staff semistructured interviews and qualitative analysis. Nurses are comfortable with patients using mHealth technology in the inpatient setting. Facilitators for the introduction of technology to hospitalized patients were identified. Based on the formative evaluation findings, we developed an Implementation Program for mHealth technology introduction in the inpatient setting.
\end{abstract}

\section{Introduction}

Electronic and mHealth technology for health behavior change has developed at rapid speeds in the last decade [1]. Enabling patients with chronic disease to benefit from these technologies is a present concern, with leaders in healthcare calling for accessible, relevant, affordable and timely technology [2]. Short messaging systems (SMS) are a promising solution, where messages can be sent to a patient at point-of-need and can be viewed by a patient on their mobile phone. It is estimated that $91 \%$ of people in the US and around $75 \%$ of the world currently have mobile phones [3-5]. While powerful apps for smart phones are continually developed, text-enabled phones are currently more highly accessible and widely utilized.

Evaluations of patient access to and utilization of technology in the inpatient setting are infrequent. In Norway, $99 \%$ of patients with coronary artery disease $(\mathrm{CAD})$ were reported to have owned a phone, while almost $63 \%$ used SMS in the hospital setting [6]. The asynchronous nature of SMS messages allows for later consideration of the messages by hospitalized patients, with patient control over the degree of exposure to the messages potentially assisting in behavior change [6].

While SMS used for tobacco cessation are publically available, there are several barriers that limit their reach. One such program, SmokefreeTXT, requires an initial online enrollment process, which is a barrier for clinicians and patients alike. QUITWORKS is another publically available telephone counseling program that targets smokers in the clinical setting for counseling after their visit or hospital stay, yet relies on clinical staff to use a fax machine to enroll patients. Patients who are enrolled in QUITWORKS are introduced to a SMS system, Text2Quit, during the initial phone call. However, due to low rates of people answering this call, many do not get the opportunity to hear about the Text2Quit program [7].

Hospitalization is a critical point in a patient's health trajectory; a teachable moment, where patients and their family members examine their health choices and are more likely to change health behaviors that impact their diagnosis [8]. Inpatient care is a window of opportunity for physicians and nurses to motivate and assist patients in making health behavior changes while under their care. Smoking is the number one health behavior that 
patients can change to improve their health outcomes and is very common among patients admitted to the hospital [9]. Hospitalization is a unique opportunity for patients to quit smoking. Involuntary abstinence and easy access to nicotine replacement therapy provide the ideal environment to make this lifestyle change. Most smokers return to smoking when discharged [10-11], and smokers who resume smoking are more likely to be rehospitalized than those who quit [12]. Helping smokers quit during hospitalization will save lives and reduce costs [1314].

The Technology Inpatient Program for Smokers (TIPS) uses motivational messages administered via SMS to hospitalized patients at-risk for poor outcomes related to tobacco use. We developed these messages using current guidelines [15], and social cognitive theory [16], and evaluated them through a web-assisted tobacco intervention (R21-CA089011, 1R01CA129091-01) [17-20]. In the outpatient setting, the motivational messages resulted in increased engagement in the online smoking intervention and increased six-month smoking cessation outcomes (odds ratio 1.7) compared with controls. In this paper, we examine processes of extending implementation of TIPS into the hospital setting. Patients using their mobile phones in the hospital will greatly reduce the barriers to enrollment in publically available SMS for tobacco cessation, without the need for another person, another device or Internet access.

Nurses are key stakeholders in health promotion and interventions during hospitalization. Nurse introduction of technology during inpatient stays will allow for in-person assistance and education to patients who may be eligible but hesitant to sign up for the program. Interventions involving nurses for patient tobacco cessation in the inpatient setting have been shown to be effective [21-22], yet are not easily integrated into usual care. As insufficient time and a lack of administrative resources are known to be the largest barriers to nurse practice change in the clinical setting, and specifically to tobacco cessation counseling by nurses [23-24], ease of introduction and patient sign-up was valued in the creation of TIPS. Commonly, nurseintroduced technologies for patients are institutionally owned devices loaned to patients for one-time use, and are either for educational or assessment purposes [25-26], or for symptom monitoring post-hospitalization with use over time [27]. As far as the authors are aware, nurses introducing the use of a technology on the patient's own device for repeated exposure to content and to increase motivation for behavior change has not been examined.

This paper describes (1) the formative evaluation prior to implementation of TIPS and (2) the adapted mHealth intervention based on the formative evaluation. Research questions for the formative evaluation include:

What is the current use of technology by patients and nurses in an inpatient setting?

What are the perceptions of comfort and appropriateness by nurses on assisting to implement an mHealth intervention for tobacco cessation in an inpatient floor?

What are the barriers to and facilitators of implementing new technology intervention for behavior change in the inpatient setting?

The discussion will address findings pertinent to implementing the introduction of mHealth technology in the inpatient setting, as well as outlining the development of components and strategies for implementation of TIPS.

\section{Methods}

Formative evaluation of the TIPS implementation program was performed. For the purpose of this paper, we will report the results of the formative evaluation and discuss the refinement of the TIPS implementation program.

\subsection{Study design}

In technology implementation, a formative evaluation with the goal of adapting protocols to a local context enhances success [28]. This formative evaluation used the Quality Enhancement Research Initiative (QUERI) Implementation Guide [29] to construct its research questions, which closely reflect formative evaluation goals of (1) describing current practice, (2) describing potential barriers and facilitators to the adoption of the new intervention, and (3) assessing perceived feasibility and utility of the project by stakeholders. Using a grounded theory approach for the qualitative analysis, nurse interviews were analyzed to identify factors that affect TIPS program implementation, based on pragmatic, implementation-focused research questions.

\subsection{Formative evaluation methods}

An interview guide was developed to identify factors that affect the implementation of a technology intervention introduced by nurses on the 
Table 1. Demographic and Clinical Practice Characteristics of Formative Evaluation Nurse Interview Participants

\begin{tabular}{|c|c|c|c|c|c|c|}
\hline & \multirow{2}{*}{\multicolumn{2}{|c|}{$\begin{array}{l}\text { All Nurses } \\
\mathrm{N}=24\end{array}$}} & \multicolumn{4}{|c|}{ All Nurses } \\
\hline & & & \multicolumn{2}{|c|}{$\begin{array}{c}\text { Nurse Leaders } \\
\mathrm{N}=8(33.3 \%)\end{array}$} & \multicolumn{2}{|c|}{$\begin{array}{c}\text { Nurse Staff } \\
\mathrm{N}=16(66.7 \%)\end{array}$} \\
\hline \multicolumn{3}{|l|}{ Demographic Characteristics } & & & & \\
\hline & $\mathbf{N}$ & $\%$ & $\mathbf{N}$ & $\%$ & $\mathbf{N}$ & $\%$ \\
\hline Age (years), mean (range) & \multicolumn{2}{|c|}{$42.7(24-64)$} & \multicolumn{2}{|c|}{$54.4(35-64)$} & \multicolumn{2}{|c|}{$36.5(24-60)$} \\
\hline \multicolumn{7}{|l|}{ Gender } \\
\hline Male & 2 & 8.3 & 0 & 0 & 2 & 12.5 \\
\hline Female & 22 & 91.7 & 8 & 100 & 14 & 87.5 \\
\hline \multicolumn{7}{|l|}{ Education } \\
\hline $\mathrm{AD}$ or $\mathrm{LPN}^{\mathrm{a}}$ & 4 & 16.7 & 0 & 0 & 4 & 25.0 \\
\hline Bachelors of Science in Nursing & 12 & 50.0 & 1 & 12.5 & 11 & 68.8 \\
\hline Masters of Science in Nursing & 7 & 29.2 & 6 & 75.0 & 1 & 6.2 \\
\hline Doctoral Degree in Nursing & 1 & 4.2 & 1 & 12.5 & 0 & 0 \\
\hline \multicolumn{7}{|l|}{ Smoking status } \\
\hline Never smoker & 15 & 62.5 & 5 & 62.5 & 10 & 62.5 \\
\hline Current smoker & 1 & 4.2 & 0 & 0 & 1 & 6.2 \\
\hline Former smoker & 8 & 33.3 & 3 & 37.5 & 5 & 31.2 \\
\hline \multicolumn{7}{|l|}{ Clinical Practice Characteristics } \\
\hline & \multicolumn{2}{|c|}{ mean (range) } & \multicolumn{2}{|c|}{ mean (range) } & \multicolumn{2}{|c|}{ mean (range) } \\
\hline Years of nurse experience & \multicolumn{2}{|c|}{$14.1(1.5-41)$} & \multicolumn{2}{|c|}{$22.8(10-41)^{b}$} & \multicolumn{2}{|c|}{$10.2(1.5-32)$} \\
\hline Work hours per week & \multicolumn{2}{|c|}{$40.2(10-80)$} & \multicolumn{2}{|c|}{$62.8(50-80)^{b}$} & \multicolumn{2}{|c|}{$30.2(10-40)$} \\
\hline Maximum patient load per shift & \multicolumn{2}{|c|}{-} & \multicolumn{2}{|c|}{-} & \multicolumn{2}{|c|}{$4.5(4-5)$} \\
\hline
\end{tabular}

hospital floor. The purpose of the formative evaluation interviews was to assist in the development of materials for the Implementation Program and refinement of its components. Qualitative analyses of these interviews will further inform the Implementation Program. The University of Massachusetts Medical School Institutional Review Board approved this study.

Key stakeholders in the implementation of the TIPS program were invited for an interview. Nurse leaders, nurse managers and nurse staff from implementation sites were invited to interview, targeting 1-3 nurse leaders from each site, 1 nurse manager from each floor and 4 staff nurses from each floor. The number of nurse leaders, managers and staff were purposefully selected to gain a range of opinions related to unique attributes to each hospital facility and floor and to achieve qualitative theme saturation [30]. Nurses who were interested were given a fact sheet and scheduled for an in-person interview, with recruitment lasting during site visits until targeted numbers were reached.

Semi-structured interviews were conducted with eight nurse leaders (four hospital leaders and four nurse managers) and sixteen nurse staff during multiple site visits by a nurse researcher experienced in interviewing nurse staff $(\mathrm{AB})$. Audio recordings were transcribed and analyzed using content analysis methods with NVIVO software for thematic content [31]. Theme saturation was reached for staff nurses, with low discovery of new facilitators and barriers to technology introduction in the inpatient setting. The themes from nurse managers and nurse leaders were convergent, and there were a low number of new themes at the conclusion of the analysis of these key stakeholders' interviews. Nodes were summarized in a table organized by research question and expanded upon in text form.

\section{Results}

Of the 24 nurses interviewed (Table 1), the majority were young (median age of 37 years), Caucasian ( $\mathrm{N}=23 ; 95.8 \%)$ women $(\mathrm{N}=22 ; 91.7 \%)$ who were bachelor's degree holders $(\mathrm{N}=12 ; 50 \%)$ and never smoked $(\mathrm{N}=15 ; 62.5 \%)$. Nurse staff $(\mathrm{N}=16)$ were young (mean 31 years), working approximately 30 hours per week and taking care of a mean of 4.5 patients per shift. Nurse leaders $(\mathrm{N}=8)$ were very experienced, with a mean of 22.8 years practicing as a nurse or in leadership, and typically worked double nurse staff hours (62.8 mean hours per week).

Nurses were eager to share their observations and perceptions of current practices, 
Table 2. Summary of Formative Evaluation Qualitative Data from Nurse Interviews

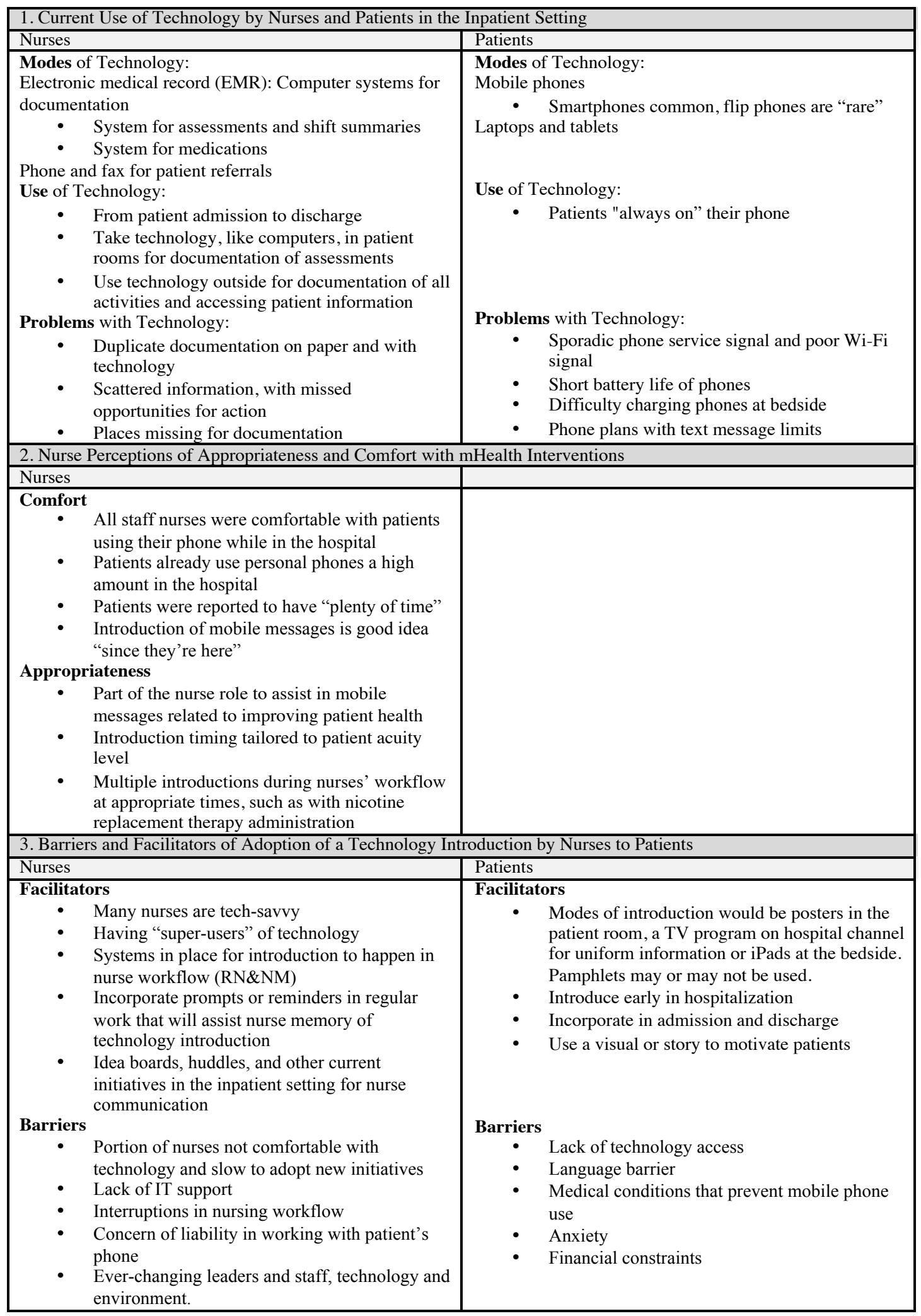


technology use, and successful initiatives in their workplace. The categories and subcategories of the main findings from the qualitative interviews are reported by research question (Table 2), with an expanded description of the main findings in the text below.

\subsection{Current use of technology}

Multiple modes of technology were available for nurses and patients to use in the inpatient setting. Computer systems for patient assessments and shift summaries, as well as a separate system for medication administration, were most common. Calling or faxing patient information for referrals was less common, and were even less likely to be utilized due to time burden compared to using the computer when online referral or messaging healthcare providers was possible. Nurses also commonly have a personal phone on which they occasionally look up pertinent medical information.

Nurses use technology from the time of patient admission to discharge, with the electronic medical record (EMR) serving as the primary repository for a large amount of patient information. An admission interview of the patient using a "flow sheet" in the EMR on a computer wheeled into the patient room is augmented by similar documentation throughout their stay, primarily medical assessments performed during and summaries at the end of each shift. This information is usually to be utilized by other healthcare team members. Immediately prior to discharge from the hospital, nurses print patient information from the EMR to be used in the outpatient setting.

In the hospital, patients use phones, laptops and tablets. Generally, nurses reported patients using a mobile phone often during their hospital stay. Problems with technology for patients included weak phone service signal, poor Wi-Fi signal for internet access, short battery life of phones, difficulty charging phones at bedside and having phone plans with limited texting service. Most patients were perceived to be "tech-savvy", except for older patients. Yet there was a sense that each patient was unique in their technology use capability: one nurse admonished, "but don't underestimate the grayhaired people," a comment stemming from her interactions with older patients who electronically tracked all of their health behaviors.

Currently, nurses cite the EMR and separate medication system as problem-prone or deficient, with a new EMR system anticipated to solve issues of documentation, tracking and reporting information. Nurse leaders noted this trend of promised improvement is similar to a system-wide rollout of the current EMR system, which is currently being replaced, and were cautiously optimistic of progressive gains in function. Nurses reported continual change in technology on patient floors. The promised future technology is seen as the solution to the currently disjointed technology systems.

\subsection{Appropriateness and comfort for introduction of technology by nurses}

Nurses believed that introduction of technology for behavior change was a part of their role. The nurses placed "assisting patients to get well" as a top priority. Introducing technology to help patients improve their health, especially patients that have chronic disease affected by their health behaviors, was aligned with the nurse role. As one staff nurse commented,

"[Signing up for a mobile health technology] is a choice for them to improve their health, and that's why I'm here is to help them get better and provide good patient care."

Generally, introduction of technology was seen as a nurse-specific action, and there was disagreement as to nursing assistant role and time available to introduce technology. However, the difficulty of the task (introducing technology) was seen as low, as one nurse stated, "it would be on the same level of difficulty as getting someone a drink of water." Nurses were also willing to accept the role of helping patients sign up for motivational texting if they are having problems, likening this to patients needing assistance restricting their fluids if warranted for their health.

Workflow integration and patient acuity considerations were identified as key factors in the appropriateness of introduction of technology. Integration into nursing workflow is key. The introduction of technology during admission is a natural part of the nurse workflow. Giving out a handout on admission would not be a normal part of the workflow on admission. Also, introducing technology during the medication pass if the patient is on NRT is also a natural part of the workflow. Since the technology assistance is seen as "quick", there is more acceptance of adoption.

Determination of appropriateness relative to patient acuity is key. Nurses feel comfortable introducing the technology intervention if it was appropriate given the patient's acuity. If the patient is sick, such as "having trouble breathing" related to their COPD or having acute pain, then it would be 
seen as inappropriate to address. However, if there is not an acute problem, then it would be appropriate. If the nurse is unable to introduce the technology to the patient because of an acute problem, then it can be added to "their list". This was likened to bringing the patient water later, as part of the list. This could happen a couple hours after admission. One nurse suggested,

"Personally speaking, during admission as long as acuity is stable enough would be a perfect time when you're asking about smoking, saying oh we have this new thing that we're piloting or group it in with that, since you're talking about smoking anyway"

All staff nurses $(\mathrm{N}=16)$ felt comfortable with patients texting under their care, with the majority stating patients currently do use their phone for texting during hospitalization. Patients were reported to have "plenty of time" and it was a good idea "since they're here". There was disagreement on patient technology savvy or ability, yet some nurses were very confident most patients were tech savvy.

"I mean, most people who have a phone can text, they're pretty tech savvy, probably more than me" (nurse staff member)

\subsection{Facilitators and barriers to technology introduction}

Nurses reported the majority of nurses as well as patients are tech-savvy, which is an important factor for nurses facilitating an introduction of healthcare technology to a patient. Several nurses were unsure how prepared some older members of their cohort were to introduce technology, and suggested having "super user" nurses with additional training to assist such nurses when needed. As a nurse leader commented,

"It seems to me that if there's a new modality out there, give it a try, and especially something that's so innovative as texting."

Current initiatives for nurse communication and education could incorporate information about technology introduction to patients, with an opportunity for nurses to learn and practice discussing healthcare technology with patients. Prompts and reminders for nurses in their daily tasks of assessment and medication administration will help them remember to determine patient need for the technology and to introduce relevant technology to patients. Information about the technology in patient rooms could include a poster on the patient's wall. Handouts for patients had mixed results, with concerns that patients do not look at or keep paper materials. Also, posters may facilitate nurse introduction, as one nurse commented,

"And I really think posters are great for some sort of information because it gives us that out to be like, 'Hey, did you think...' Without being [in scolding voice] 'Did you think about quitting smoking?' It like gives us that 'in', to bring it up, without patients feeling like we're attacking them. So then it's a little bit easier to kind of address."

Facilitation of technology introduction to patients could use other modes of technology, such as a TV program on a hospital channel in their room or iPads at the bedside. These modes could enhance uniform patient education of information across the hospital. Suggestions to use a visual aid or a story to motivate patients to sign up were given, such as having a patient with a tracheostomy address patients. Another strategy is to assess the patient's own comfort with technology and what works for them. Lastly, nurses suggested incorporating the introduction to patients early in the hospitalization.

Barriers to introduction of technology include continual change in technology, hospital floor environment, and people, including leaders and staff. There was a reported lack of IT support in the hospital for nurses or patients, with technology support for patients nonexistent. The issue of liability for damages if a patient's technology device is broken, stolen, or does not function related to the healthcare technology introduced was a concern to both nurse leaders and nurse staff. Lastly, there are nurse staff resistant and slow to adopt changes in technology or other new initiatives on the hospital floor. The most common barrier reported for patient introduction to a technology is lack of a mobile phone or access to alternative technology. Other barriers include financial constraints related to phone plans, language barriers, anxiety from being in the hospital, and medical conditions that do not allow the patient to use technology while in the hospital setting.

\section{Discussion}

Using the results of the formative evaluation to influence the development of an mHealth technology for tobacco cessation in the inpatient setting, lessons learned from each research question were incorporated into the Implementation Program components and strategies for inpatient adoption. 
Current technology use in the hospital setting consisted primarily of nurses using an electronic medical record (EMR) and patients using their mobile phones. Lessons learned about current technology use include:

(1) Using the EMR as a tool to remind and solidify the introduction of technology in the nurses' routine for patient care was essential. The introduction of technology needs to be integrated into this system to remind nurses during the admission interview and assessment, avoiding the use of paper materials or the need to fax materials that are interruptions to nurse routines.

(2) Identifying a specific place in the EMR for documentation of patient teaching performed is important. This could be in free-text nursing shift assessments and shift summaries. Finding a place for free-text for patient education and technology introduction is key for communicating to the rest of the team.

(3) Using patient phones to administer the technology intervention may reach many patients. Patients already commonly use a phone for text messaging while in the hospital. Patients can choose whether they desire to receive health-related text messages or if their phone plan will support these messages when the technology is introduced to them. There is still alternative educational support for patients in the hospital setting if a patient cannot use the technology, such as tobacco cessation counseling. Similarly, having this alternative is important because uptake of TIPS may be different across different ages. However, rates of text-enabled phones in the U.S. are now quite high and are expected to continue to increase toward universality.

Nurse perceptions of appropriateness of technology introduction to patients led to the identification of this action as part of the nurse role. The lessons learned are expanded upon below and will be used to assist in activating nurses in technology introduction.

(1) Nurse's view the introduction of technology to patients for the sake of patient education and behavior change as a part of their role and scope of practice. Nurses are aware of many patients already using SMS on their personal phones during hospitalization. Nurses would take ownership of this initiative since it is seen as a part of their role and scope of practice.

(2) Nurses would introduce the technology to patients during their hospitalization as a part of routine tasks like assessment and medication administration, as deemed appropriate related to the patient's acuity and present concerns.
The facilitators and barriers to technology introduction ascertained in this study point to three key lessons for implementation, including a visual cue for technology introduction, support for nurse leaders and activating nurses to introduce technology.

(1) Creating and providing a visual aid and message for patients to motivate them to sign up for messages, without using handouts.

(2) Supporting nurse leaders by tailoring support of the technology introduction by floor, such as having the nurse manager identify "super-users" of the technology to assist in the initiation of introductions by nurse staff, will be vital.

(3) Activating nurse staff to introduce technology by familiarizing them with training using current floor initiatives or meeting times (which can be identified by nurse managers) will be key. Incorporating the introduction of technology into nurse workflow and treating it as patient education for the health behavior it seeks to affect will be important.

These collected perceptions provided the structure for the Implementation Program strategy development.

\subsection{Implementation Program Design}

Based on lesssons learned from the formative evaluation, we have refined an implementation program called Technology Inpatient Program for Smokers (TIPS) (Figure 1). The implementation program (IP) components include:

1. Posters with mHealth messaging sign-up information in patient rooms

2. Nurse-administered introduction to the mobile messages

3. Tobacco Treatment Specialist introduction to the mobile messages

Tobacco Treatment Specialists (TTS) are employed by the hospital to assist current tobacco users to quit smoking, and are willing stakeholders in the introduction of technology for tobacco cessation as a part of their usual care. The effectiveness of both TTS and nurse introduction of the mobile messages relative to nurse-only introduction will be determined.

Implementation strategies are employed to influence the success of the implementation program components. Strategies are organized by lessons learned about facilitators for implementation of technology from the formative evaluation results. Strategy 1 (supporting IP components 1 and 2) is to support nurse managers as change agents, while identifying and addressing barriers to implementation as they are identified. Strategy 2 (supporting IP component 1) is the design and supply of the posters 


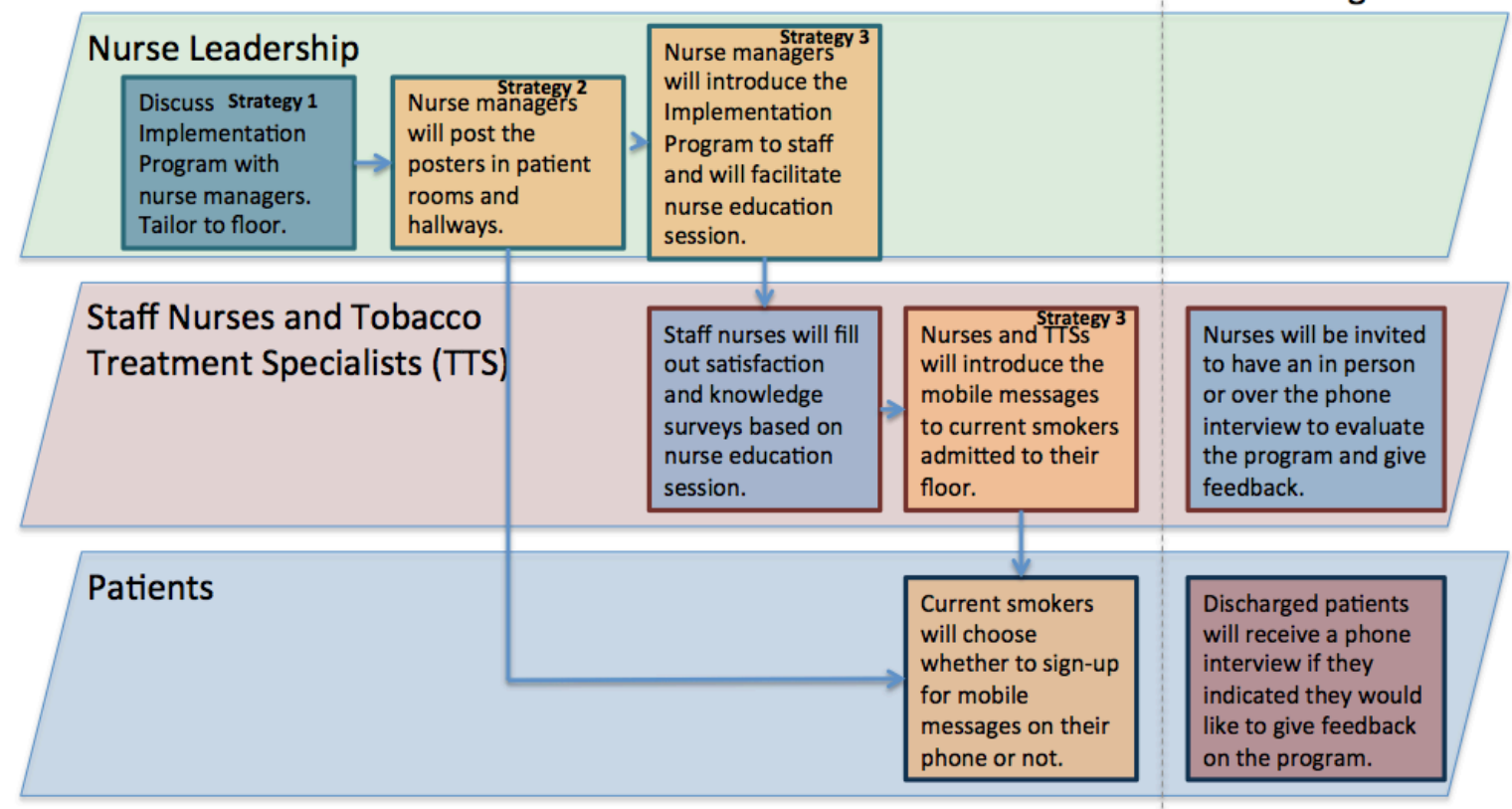

Figure 1. Developed Implementation Program from Formative Evaluation; the Technology Inpatient Program for Smoking (TIPS)

to the nurse managers, as well as identification of support for poster hanging. Strategy 3 (supporting IP components 2 and 3 ) is training and mobilizing nurses and tobacco treatment specialists to introduce the mobile messages to patients on their floor.

4.1.1. Strategy 1: support nurse leaders. An initial and ongoing discussion with nurse leaders and managers on each hospital floor about floor-specific needs to facilitate the use of the Implementation Program will be conducted. Weekly discussions will be conducted with nurse managers, touching upon aspects of the floor that facilitate or are potential barriers to introducing the mobile messages to inpatient tobacco users. Pragmatic flexibility will be given to the nurse manager's choices about means of interaction (in-person, over the phone, over email), length of interaction, and content of discussions, outside of core questions related to barriers, facilitators and material needs. Frequency of interactions (every week) will remain fixed.

\subsubsection{Strategy 2: provide posters to nurse leaders.} We designed a low-cost poster with an invitation for patients who are current smokers on the hospital floor to sign-up to receive motivational mobile messages intended to enable tobacco cessation. These posters will be hung by nurse leaders and managers, with the help of other staff, in patient rooms and in floor hallways as appropriate.
The design of the poster used the Health Belief Model and guidelines for movie poster construction. The behavior targeted for change is patient sign-up for the intervention using their phone (not to help them stop smoking). The Health Belief Model uses individual's perceptions of how threatening smoking is to them, the severity of their problem, and the benefits and barriers to taking the action of signing up for TIPS [32]. Cues to action are given with the message "Sign up by texting [phone number]" in large print, and self-efficacy was addressed by identifying the messages as "smoker-written," in an effort for the patient to identify with the message. Movie poster guidelines were used to inform layout. Importance of "actors" was conveyed using placement, with a patient and nurse examining a mobile phone in the middle, with an action of assistance by the nurse for the patient. A logline, a compelling phrase informing on content, was added.

Supply and support of floor-specific needs for nurse managers will be supported. Nurse managers will identify needs for hanging posters, including poster size and desired materials for fixing the posters to walls. The research team will supply the nurse manager with a poster for every bed, hallway and waiting room, as well as additional requested materials. Nurse managers may identify staff to assist with hanging the posters. 
4.1.3. Strategy 3: nurse and tobacco treatment specialist introduction. Nurse staff will receive an introduction to the mobile messaging system by the nurse manager. A nurse education session has been developed to train nurses and tobacco treatment specialists before they are mobilized to action in the study.

A nurse education session was developed using the ADDIE model. This model is a "systematic approach to instructional development," progressing through stages of analysis, design, development, implementation and evaluation, and was used as an instructional design tool to structure the education sessions [33]. Nurse managers and nurse staff will receive a nurse education session related to tobacco use, treatment, behavioral intervention and case studies related to introducing the mobile messages to patients. This educational session material has been used and tested before in previous hospital tobacco cessation studies in the VA Medical Centers [34]. Author permission to use the content of the slides has been obtained, and they have been modified using ADDIE to fit the goals of this study. Tobacco treatment specialists will assist the nurse manager in administering the nurse education session to nurse staff, due to their expertise in addressing tobacco cessation with patients and familiarity with the mobile message system.

A nurse and tobacco treatment specialist introduction protocol was developed. Nurses on the floors and a tobacco treatment specialist employed by the hospital will introduce patients to mobile messages. Nurses and tobacco treatment specialists have introduced a variety of tobacco treatment interventions to patients in the hospital setting but have not previously introduced mobile messages.

\section{Conclusion}

Utilizing facilitators and addressing barriers for nurses to introduce the mHealth intervention for implementation in the inpatient setting, we developed an Implementation Program for mHealth technology introduction in the inpatient setting. Nurses are key stakeholders in the introduction of health technology introduction for patients in the hospital setting. Nurse staff are comfortable with patients using their mobile phones for SMS while under their care. Nurse leaders and staff recognize mHealth technology introduction for improvement of patient health as a task relevant to the nurse role, and it is considered well suited to fit in the current nurse work routine.

\section{References}

[1] R. Orji, K. Moffatt, "Persuasive technology for health and wellness: State-of-the-art and emerging trends", Health Informatics J, 2016, Epub.

[2] Healthy People 2020. (2014). Tobacco use [Online]. Available https://www.healthypeople.gov/2020/topicsobjectives/topic/tobacco-use

[3] P.M. Vernig, R.J. Repique, "Short message service can be a promising tool for psychiatric patients and clinicians", J Am Psychiatr Nurses Assoc, 21 (2015), pp. 31-33.

[4] A. Beratarrechea, A.G. Lee, J.M. Willner, E. Jahangir, A. Ciapponi, A. Rubinstein, "The impact of mobile health interventions on chronic disease outcomes in developing countries: a systematic review", Telemed J E Health, 20 (2014), pp. 75-82.

[5] World Bank. (2012, July 17). Mobile phone access reaches three quarters of planet's population [Online]. Available http://www.worldbank.org/en/news/pressrelease/2012/07/17/mobile-phone-access-reaches-threequarters-planets-population

[6] S. Bergvik, R. Wynn, "The use of short message service (SMS) among hospitalized coronary patients", Gen Hosp Psychiatry, 34 (2012), pp. 390-397.

[7] Willett, J. G., Hood, N. E., Burns, E. K., Swetlick, J. L., Wilson, S. M., Lang, D. A., and Levinson, A. H. (2009). "Clinical faxed referrals to a tobacco quitline: reach, enrollment, and participant characteristics." Am J Prev Med, 36(4), 337-340.

[8] B. Dohnke, C. Ziemann, K.E. Will, E. Weiss-Gerlach, C.D. Spies, "Do hospital treatments represent a 'teachable moment' for quitting smoking? A study from a stagetheoretical perspective", Psychol Health, 27 (2012), pp. 1291-1307.

[9] G. Danaei, E.L. Ding, D. Mozaffarian, B. Taylor, J. Rehm, C.J. Murray, M. Ezzati, "The preventable causes of death in the United States: comparative risk assessment of dietary, lifestyle, and metabolic risk factors", PLoS Med, 6 (2009), e1000058.

[10] A.N. Thorndike, N.A. Rigotti, "A tragic triad: coronary artery disease, nicotine addiction, and depression”, Curr Opin Cardiol, 24 (2009), pp. 447-453.

[11] M.F. Attebring, M. Hartford, A. Hjalmarson, K. Caidahl, T. Karlsson, J. Herlitz, "Smoking habits and predictors of continued smoking in patients with acute coronary syndromes", J Adv Nurs, 46 (2004), pp. 614-623.

[12] D. Hasdai, K.N. Garratt, D.E. Grill, A. Lerman, D.R. Holmes, "Effect of smoking status on the long-term outcome after successful percutaneous coronary revascularization”, N Engl J Med, 336 (1997), pp. 755-761. 
[13] N.A. Rigotti, M.R. Munafo, L.F. Stead, "Interventions for smoking cessation in hospitalized patients, Cochrane Database Syst Rev", (2007) CD001837.

[14] N.A. Rigotti, M.R. Munafo, L.F. Stead, Smoking cessation interventions for hospitalized smokers: a systematic review, Arch Intern Med, 168 (2008), pp. 19501960.

[15] M. Fiore, C. Jaen, T. Baker, et al., Treating Tobacco Use and Dependence: 2008 Update, Clinical Practice Guideline, U.S. Department of Health and Human Services. Public Health Service, Rockville, MD, 2008.

[16] A. Bandura, Social Foundations of Thought and Action: a Social Cognitive Theory, Prentice Hall, Englewood Cliffs, NJ, 1986.

[17] T.K. Houston, R.S. Sadasivam, J.J. Allison, A.S. Ash, M.N. Ray, T.M. English, T.P. Hogan, D.E. Ford, "Evaluating the QUIT-PRIMO clinical practice ePortal to increase smoker engagement with online cessation interventions: a national hybrid type 2 implementation study”, Implement Sci, 10 (2015) 154.

[18] T.K. Houston, R.S. Sadasivam, D.E. Ford, J. Richman, M.N. Ray, J.J. Allison, "The QUIT-PRIMO providerpatient Internet-delivered smoking cessation referral intervention: a cluster-randomized comparative effectiveness trial: study protocol", Implement Sci, 5 (2010), pp. 87.

[19] T.K. Houston, J.S. Richman, M.N. Ray, J.J. Allison, G.H. Gilbert, R.M. Shewchuk, C.L. Kohler, C.I. Kiefe, D.C. Group, "Internet delivered support for tobacco control in dental practice: randomized controlled trial", J Med Internet Res, 10 (2008), e38.

[20] R.S. Sadasivam, K. Delaughter, K. Crenshaw, H.J. Sobko, J.H. Williams, H.L. Coley, M.N. Ray, D.E. Ford, J.J. Allison, T.K. Houston, "Development of an interactive, Web-delivered system to increase provider-patient engagement in smoking cessation", J Med Internet Res, 13 (2011), e87.

[21] S.A. Duffy, D.L. Ronis, C.A. Karvonen-Gutierrez, L.A. Ewing, G.W. Dalack, P.M. Smith, T.P. Carmody, T. Hicks, C. Hermann, P. Reeves, P. Flanagan, "Effectiveness of the tobacco tactics program in the Department of Veterans Affairs", Ann Behav Med, 48 (2014), pp. 265274.

[22] A.M. Fore, C.A. Karvonen-Gutierrez, A. Talsma, S.A. Duffy, 'Nurses' delivery of the Tobacco Tactics intervention at a Veterans Affairs Medical Center", J Clin Nurs, 23 (2014), pp. 2162-2169.

[23] Melnyk, B. M., Fineout-Overholt, E., Gallagher-Ford, L., and Kaplan, L. (2012). "The state of evidence-based practice in US nurses: critical implications for nurse leaders and educators." J Nurs Adm, 42(9), 410-417.
[24] Li, I. C., Lee, S. Y., Chen, C. Y., Jeng, Y. Q., and Chen, Y. C. (2014). "Facilitators and barriers to effective smoking cessation: counselling services for inpatients from nurse-counsellors' perspectives--a qualitative study." Int J Environ Res Public Health, 11(5), 4782-4798.

[25] Sawyer, T., Nelson, M. J., McKee, V., Bowers, M. T., Meggitt, C., Baxt, S. K., Washington, D., Saladino, L., Lehman, E. P., Brewer, C., Locke, S. C., Abernethy, A., Gilliss, C. L., and Granger, B. B. (2016). "Implementing Electronic Tablet-Based Education of Acute Care Patients." Crit Care Nurse, 36(1), 60-70.

[26] Schuler, M. K., Trautmann, F., Radloff, M., Schmädig, R., Hentschel, L., Eberlein-Gonska, M., Petzold, T., Vetter, H., Oberlack, S., Ehninger, G., and Schmitt, J. (2016). "Implementation of a mobile inpatient quality of life (QoL) assessment for oncology nursing." Support Care Cancer, 24(8), 3391-3399.

[27] Ritchie, C. S., Houston, T. K., Richman, J. S., Sobko, H. J., Berner, E. S., Taylor, B. B., Salanitro, A. H., and Locher, J. L. (2016). "The E-Coach technology-assisted care transition system: a pragmatic randomized trial." Transl Behav Med, 6(3), 428-437.

[28] C.B. Stetler, M.W. Legro, C.M. Wallace, C. Bowman, M. Guihan, H. Hagedorn, B. Kimmel, N.D. Sharp, J.L. Smith, "The role of formative evaluation in implementation research and the QUERI experience", J Gen Intern Med, 21 Suppl 2 (2006), pp. S1-8.

[29] Quality Enhancement Research Initiative. (2014). QUERI Implementation Guide [Online]. Available: www.queri.research.va.gov/implementation/Implementatio nGuide_4.pdf

[30] J.W. Creswell, Qualitative inquiry and research design: Choosing among five traditions, Sage, Thousand Oaks, CA, 1998.

[31] J. Saldana, The Coding Manual for Qualitative Researchers, Sage, Thousand Oaks, CA, 2012.

[32] K. Glanz and B.K. Rimer, Theory at a Glance: A Guide for Health Promotion Practice ( $2^{\text {nd }}$ ed.), National Cancer Institute, National Institutes of Health, U.S. Department of Health and Human Services, NIH Pub, Washington, DC, 2005.

[33] M. Molenda. (2003). ADDIE Model [Online]. Available:http://iptde.boisestate.edu/FileDepository.nsf/bf2 5ab0f47ba5dd785256499006b15a4/693b43c6386707fc 872 578150059c1f3/\%24FILE/Molenda_03.pdf

[34] S.A. Duffy, D.L. Ronis, M.G. Titler, F.C. Blow, N. Jordan, P.L. Thomas, G.L. Landstrom, L.A. Ewing, A.H. Waltje, "Dissemination of the nurse-administered Tobacco Tactics intervention versus usual care in six Trinity community hospitals: study protocol for a comparative effectiveness trial", Trials, 13 (2012), pp. 125. 